PROCEEDINGS OF THE

AMERICAN MATHEMATICAL SOCIETY

Volume 126, Number 7, July 1998, Pages 2089-2094

S 0002-9939(98)04202-6

\title{
MEAN EXIT TIME FROM CONVEX HYPERSURFACES
}

\author{
VICENTE PALMER
}

(Communicated by Peter Li)

\begin{abstract}
L. Karp and M. Pinsky proved that, for small radius $R$, the mean exit time function $E_{R}$ of an extrinsic $R$-ball in a hypersurface $P^{n-1} \subseteq \mathbb{R}^{n}$ is bounded from below by the corresponding function $\widetilde{E}_{R}$ defined on an extrinsic $R$-ball in $\mathbb{R}^{n-1}$. A counterexample given by C. Mueller proves that this inequality doesn't holds in the large. In this paper we show that, if $P$ is convex, then the inequality holds for all radii. Moreover, we characterize the equality and show that analogous results are true in the sphere.
\end{abstract}

\section{INTRODUCTION}

Lower bounds for certain riemannian invariants like the mean exit time of Brownian motion or the volume of extrinsic balls of submanifolds in a riemannian manifold had been given by several authors (see [ChYL], [Ma1],[Ma2], [KP1], [KP2]).

In [KP1] the authors get an asymptotic formula for the mean exit time function $E_{R}$ of an extrinsic ball of small radius $R$ in a hypersurface $P$ of $\mathbb{R}^{n}$, in terms of the mean curvature of $P$. This fact leads to characterizations of minimal hypersurfaces using the mean exit time function ([KP1] and [Ma1]).

When the hypersurface is not minimal, it follows from the asymptotic expansion found in [KP1] that $E_{R} \geq \frac{R^{2}-r^{2}}{2(n-1)}$, for $R$ sufficiently small. So it is natural to wonder if this inequality holds for every $R$, when $P$ is complete. In general, the answer is no, and a counterexample was given by $\mathrm{C}$. Mueller in $[\mathrm{M}]$ consisting of a very thin tube which has strongly negative curvature.

In this paper we show (Theorem 1) that the inequality above holds, for any radius, when $P$ is a complete convex hypersurface of $\mathbb{R}^{n}$ (hence, a positively curved manifold). Moreover, we extend the same result for convex hypersurfaces of the sphere $S^{n}(1)$, and characterize the equality in both cases.

In order to state our results, we shall introduce some notation and terminology.

Let $P^{n-1}$ be an orientable, embedded complete hypersurface of the riemannian manifold $\mathbb{K}^{n}(\lambda)$. Throughout this paper, $\mathbb{K}^{n}(\lambda)$ is taken to be one of the simply connected space forms with curvature $\lambda=0$ or 1 , i.e. $\mathbb{K}^{n}(\lambda)=\mathbb{R}^{n}$ or $S^{n}(1)$ respectively.

Received by the editors August 6, 1996 and, in revised form, December 10, 1996.

1991 Mathematics Subject Classification. Primary 53C21, 58G32.

Key words and phrases. Brownian motion, mean exit time, convex hypersurface, extrinsic ball. Work partially supported by a DGICYT Grant No. PB94-0972.

(C)1998 American Mathematical Society 
The distance function on the ambient space $\mathbb{K}^{n}(\lambda)$ is denoted by $d$, so, if $p \in P$, we can define $r(q):=d(p, q)$ for every $q \in \mathbb{K}^{n}(\lambda)$. We shall also denote by $r$ the restriction $\left.r\right|_{P}: P \longrightarrow \mathbb{R}$. This restriction is called the extrinsic distance to $p$ in $P$.

Let us define the extrinsic ball of radius $R$ and center $p \in P, D_{R}(p) \subseteq P$, to be the smooth connected component of $B_{R}^{\lambda}(p) \cap P=\left\{q \in P / r_{p}(q) \leq R\right\}$ which contains $p$. Here, $B_{R}^{\lambda}(p)$ denotes the geodesic $R$-ball around $p$ in the ambient space $\mathbb{K}^{n}(\lambda)$. (From now on, when $\lambda=1, R \leq \frac{\pi}{2}$.)

The extrinsic ball $D_{R}(p)$ is a connected and compact domain in $P$, with boundary

$$
\partial D_{R}(p) \subseteq\{q \in P / r(q)=R\}=\left\{q \in \mathbb{K}^{n}(\lambda) / r_{p}(q)=R\right\} \cap P=S_{R}^{\lambda}(p) \cap P
$$

where $S_{R}^{\lambda}(p)$ denotes the geodesic sphere of radius $R$ around $p$ in $\mathbb{K}^{n}(\lambda)$.

Let us observe that, when we consider the totally geodesic hypersurfaces $\mathbb{P}_{\lambda}=$ $\mathbb{R}^{n-1}$, or $S^{n-1}(1)$ in $\mathbb{K}^{n}(\lambda)=\mathbb{R}^{n}$, or $S^{n}(1)$ respectively, then the corresponding extrinsic $R$-balls centered at $\tilde{p} \in \mathbb{P}_{\lambda}, D_{R}^{\lambda}(\tilde{p})$, will be the geodesic $R$-balls centered at $\tilde{p}$ in these hypersurfaces. We shall refer them to be the standard situations.

On the other hand, we shall denote by $\partial_{r}$ and $\partial_{t}$ the corresponding gradients of $r$ in $\mathbb{K}^{n}(\lambda)$ and $P$ respectively. Let us remark that $\partial_{t}(q)$ is just the tangential component in $P$ of $\partial_{r}(q)$, for all $q \in P$. Then, if $\xi$ denotes the unit normal to $P$, we have the following basic relation on $\partial D_{R}(p)$, for all $R$ : (see [JK, eq. (2.1)])

$$
\partial_{r}=\left\langle\partial_{r}, \xi\right\rangle \xi+\partial_{t} .
$$

The problem we have considered arises from the study of Brownian motion on the domain $D_{R}(p)$. If we denote by $E_{R}(x)$ the mean time of first exit from $D_{R}(p)$ for a Brownian particle starting at $x \in D_{R}(p)$, a remark due to Dynkin ([Dy]) states that $E_{R}$ verifies the Poisson Equation with Dirichlet boundary data:

$$
\begin{aligned}
\Delta E_{R} & =-1, \\
\left.E_{R}\right|_{\partial D_{R}} & =0 .
\end{aligned}
$$

We want to compare $E_{R}$ with the mean exit time function $\widetilde{E}_{R}^{\lambda}(\tilde{x})$ defined on the extrinsic $R$-ball $D_{R}^{\lambda}(\tilde{p})$ in the standard cases. In these standard situations, $D_{R}^{\lambda}(\tilde{p})$ has maximal isotropy at the center $\tilde{p}$, so we have that $\widetilde{E}_{R}^{\lambda}$ only depends on the extrinsic distance of $\tilde{x}$ from $\tilde{p}$. Therefore, we will write $\widetilde{E}_{R}^{\lambda}(\tilde{x})=\widetilde{E}_{R}^{\lambda}(r)$. In order to compare $E_{R}$ and $\widetilde{E}_{R}^{\lambda}$, we transplant $\widetilde{E}_{R}^{\lambda}$ to $D_{R}(p)$ by the following definition:

$$
E_{R}^{\lambda}: D_{R}(p) \longrightarrow \mathbb{R} ; E_{R}^{\lambda}(x):=\widetilde{E}_{R}^{\lambda}\left(r_{p}(x)\right) .
$$

Our result can now be formulated as follows.

Theorem 1. Let $P^{n-1}$ be a complete convex hypersurface of $\mathbb{R}^{n}$ or $S^{n}(1)$. Then, for each $R$ and $\lambda=0,1$

$$
\begin{array}{r}
E_{R}^{\lambda}(x) \leq E_{R}(x) \forall x \in D_{R}(p) . \\
\text { If } E_{R}^{\lambda}(x)=E_{R}(x) \quad \forall x \in D_{R}(p) \text {, then } D_{R}(p)=D_{R}^{\lambda} .
\end{array}
$$

We shall prove Theorem 1 in $\S 3$. $\S 2$ is devoted to some preliminary computations and a description of some fundamental facts about convexity of hypersurfaces.

This work was partially done during a stay at the Centre de Recerca Matematica (Institut d'Estudis Catalans/Universitat Autonoma de Barcelona), supported by a Grant of the Conselleria de Educacio i Ciencia de la Generalitat Valenciana.

We wish to thank Vicente Miquel for his advice and helpful comments. 


\section{Preliminaires}

Let $f: P \longrightarrow \mathbb{R}$ be a function that depends only on the extrinsic distance $r$. Then $f(r)$ will denote the composition $f \circ r: P \longrightarrow \mathbb{R}$. Following [GW] and [JK], we have that

$$
\Delta^{P} f=f^{\prime \prime}(r)\left\|\partial_{t}\right\|^{2}+f^{\prime}(r) \Delta^{P} r
$$

where $\Delta^{P}$ denotes the laplacian on $P$.

When $P$ lies in a space form $\mathbb{K}^{n}(\lambda)$, it can be proved that (see [Ma1],[Ma2] and $[\mathrm{CGM}])$

$$
\Delta^{P} r=-h_{\lambda}(r)\left\|\partial_{t}\right\|^{2}+(n-1)\left(h_{\lambda}(r)+\left\langle H, \partial_{r}\right\rangle\right)
$$

and

$$
\Delta^{P} f=\left(f^{\prime \prime}(r)-f^{\prime}(r) h_{\lambda}(r)\right)\left\|\partial_{t}\right\|^{2}+(n-1) f^{\prime}(r)\left(h_{\lambda}(r)+\left\langle H, \partial_{r}\right\rangle\right)
$$

where $h_{\lambda}(r)$ is the mean curvature of any geodesic sphere of radius $r$ in $\mathbb{K}^{n}(\lambda)$ and $H$ is the mean curvature vector of $P$.

We recall that, when we take the normal to the geodesic sphere in $\mathbb{K}^{n}(\lambda)$, pointing inward,

$$
h_{\lambda}(r)= \begin{cases}\frac{1}{\tan r}, & \text { if } \lambda=1, \\ \frac{1}{r}, & \text { if } \lambda=0 .\end{cases}
$$

Now, some facts about convex hypersurfaces.

2.1 Definition (see [KN, vol. II, p. 40] and [Sp, vol III, p.93]). A hypersurface $P^{n-1}$ in $\mathbb{R}^{n}$ is said to be convex if, for every $q \in P$, the tangent hyperplane of $P$ at $q, T_{q} P$, does not separate $P$ into two parts.

Convexity extends to hypersurfaces in $S^{n}(1)$ if we replace in the above definition the tangent hyperplane $T_{q} P$ by the totally geodesic hypersurface $\exp \left(T_{q} P\right)$ in $S^{n}(1)$ (see [Sp, vol IV, pp.121-123]).

2.2 Remark. (See [Sp, vol IV, pp.121-123], [Sp, vol III, pp.91-95] and [DCW].) A classical generalization of Hadamard's Theorem states that, if $P$ is a convex hypersurface in $\mathbb{R}^{n}$, then the Weingarten map of $P, L_{\xi}$, is semidefinite for all $q \in P$. Therefore, all the principal curvatures of $P$ will be non-negative or non-positive according to the orientation chosen for $P$.

The same result holds for convex hypersurfaces in $S^{n}(1)$ (see [DCW], [Sp, Vol. IV, p. 123]).

Given a set $A \subseteq \mathbb{K}^{n}(\lambda), A$ is convex if and only if $A$ contains the segment of the unique geodesic between $p$ and $q$ whenever $p, q \in A$. A convex hypersurface in $\mathbb{K}^{n}(\lambda)$ can be viewed as the boundary of a convex set in $\mathbb{K}^{n}(\lambda)$ (see [Sp]).

When $\lambda=1$, a convex hypersurface in $S^{n}(1)$ must be contained in some open hemisphere.

We are going to state a technical result which relates the convexity of hypersurfaces to the spatial position of normal vector fields $\partial_{r}$ and $\xi$, along the boundary of an extrinsic ball $\partial D_{r}(p)$ of any radius.

2.3 Proposition. Let us suppose that $P$ is a convex hypersurface in $\mathbb{K}^{n}(\lambda)$. Then we can choose the orientation of $P$ in such a way that

$$
\left\langle\xi, \partial_{r}\right\rangle(m) \geq 0 \text { for each } m \in \partial D_{r}(p) \text {, for all } r \text {. }
$$


Proof. First, let us suppose that $\lambda=0$. Since $P$ is convex, then $\mathbb{R}^{n}-P=\Omega_{1} \cup \Omega_{2}$, where $\Omega_{i}(i=1,2)$ are disjoint open sets, and one of them, say $\Omega_{1}$, is a convex subset of $\mathbb{R}^{n}$. The closure of this set $\bar{\Omega}_{1}=\Omega_{1} \cup P$ is convex too.

If $\xi$ is the unit normal vector field of $P$ at $m \in \partial B_{r}(p)$, pointing outward (that is, to $\Omega_{2}$ ), then $\bar{\Omega}_{1}$ is on the side of the tangent hyperplane to $P$ in $m$, opposite to $\xi$. On the other hand, the normal vector $\partial_{r}$ to $S_{r}^{0}(p)$ is on the straightline joining $m$ and $p$. As $m, p \in P \subseteq \bar{\Omega}_{1}$, the segment joining $m$ and $p$ lies in $\bar{\Omega}_{1}$, and therefore, $\partial_{r}$ points to the half-space bounded by $T_{m} P$ which is contained in $\bar{\Omega}_{2}$. This half-space is just the half-space containing $\xi$, so $-\frac{\pi}{2} \leq \angle\left(\partial_{r}, \xi\right) \leq \frac{\pi}{2}$, and then, $\left\langle\xi, \partial_{r}\right\rangle=\cos \left(\angle\left(\partial_{r}, \xi\right)\right) \geq 0$.

When $\lambda=1$, the proof is the same, replacing "unique straightline" by "unique geodesic", "tangent hyperplane" by "totally geodesic $\exp \left(T_{m} P\right)$ " and keeping in mind that $T_{m} \exp \left(T_{m} P\right)=T_{m} P$.

\section{Proof of Theorem 1}

We shall need some previous considerations about the standard situations.

We have pointed out in $\S .1$ that the extrinsic $R$-balls in standard situations are geodesic $R$-balls in $\mathbb{P}_{\lambda}$. In fact, the restriction of the extrinsic distance from $\tilde{p}$ in $\mathbb{P}_{\lambda}$ is just the intrinsic distance from $\tilde{p}$ because $\mathbb{P}_{\lambda}$ is totally geodesic in $\mathbb{K}^{n}(\lambda)$. From this consideration it follows too that $\partial_{r}=\partial_{t}$, and hence $\left\|\partial_{t}\right\|^{2}=1$.

(i) When $\lambda=0, \widetilde{E}_{R}^{0}$ is a radial solution of Dynkin's equation (1.2) on $D_{R}^{0}$. So, on account of the above consideration and (2.3), we have that $\widetilde{E}_{R}^{0}(r)$ satisfies

$$
\widetilde{E}_{R}^{0^{\prime \prime}}(r)+\widetilde{E}_{R}^{0^{\prime}}(r)\left(\frac{n-2}{r}\right)=-1 ; r \in[0, R]
$$

with boundary conditions

$$
\widetilde{E}_{R}^{0^{\prime}}(0)=0 ; \widetilde{E}_{R}^{0}(R)=0 .
$$

The solution of this equation is $\widetilde{E}_{R}^{0}(r)=\frac{R^{2}-r^{2}}{2(n-1)}$, and, hence,

$$
\widetilde{E}_{R}^{0^{\prime}}(r)=-\frac{r}{n-1}<0 \text { for all }=r \in[0, R]
$$

and

$$
\widetilde{E}_{R}^{0^{\prime \prime}}(r)=-\frac{1}{n-1}<0 \text { for all } r \in[0, R] .
$$

(ii) The same arguments shows that, when $\lambda=1, \widetilde{E}_{R}^{\lambda}(r)$ satisfies

$$
\widetilde{E}_{R}^{\lambda^{\prime \prime}}(r)+\widetilde{E}_{R}^{\lambda^{\prime}}(r)\left(\frac{n-2}{\tan r}\right)=-1 ; r \in[0, R]
$$

with boundary conditions

$$
\widetilde{E}_{R}^{\lambda^{\prime}}(0)=0 ; \widetilde{E}_{R}^{\lambda}(R)=0
$$

Changing the variable from $r$ to $s(r)=1-\cos r$ we define $\mathcal{E}_{R}^{\lambda}(s):=\widetilde{E}_{R}^{\lambda}(r(s))$. An easy computation gives us

$$
\begin{aligned}
\widetilde{E}_{R}^{\lambda^{\prime}}(r) & =\sin r \mathcal{E}_{R}^{\lambda^{\prime}}(s), \\
\widetilde{E}_{R}^{\lambda^{\prime \prime}}(r) & =\sin ^{2} r \mathcal{E}_{R}^{\lambda^{\prime \prime}}(s)+\cos r \mathcal{E}_{R}^{\lambda^{\prime}}(s) .
\end{aligned}
$$


It can be proved (see [Ma1, Proposition 4]) that

$$
\mathcal{E}_{R}^{\lambda^{\prime}}(s)<0 \text { and } \mathcal{E}_{R}^{\lambda^{\prime \prime}}(s)<0
$$

Now, let us consider, in the first place, the case $\lambda=0$. Let $E_{R}^{0}$ be the transplanted function on $D_{R}(p)$. Using (2.3), and in view of (i), and taking into account that $h_{0}(r)=\frac{1}{r}$, it follows that

$$
\Delta^{P} E_{R}^{0}=-1-r\left\langle H, \partial_{r}\right\rangle=\Delta^{P} E_{R}-r\left\langle H, \partial_{r}\right\rangle .
$$

By the convexity of $P$, and applying Proposition 2.3, we have

$$
\left\langle H, \partial_{r}\right\rangle(q)=-\frac{1}{n-1} \sum_{i=1}^{n-1}\left|k_{i}(q)\right|\left\langle\xi, \partial_{r}\right\rangle(q) \leq 0 \forall q \in D_{R}(p)
$$

where the $k_{i}$ are the principal curvatures of $P$.

From (3.5) and (3.6) follows that

$$
\Delta^{P} E_{R}^{0}(q) \geq \Delta^{P} E_{R}(q) \forall q \in D_{R}(p)
$$

so $E_{R}^{0}-E_{R}$ is a subharmonic function on $D_{R}(p)$, vanishing on $\partial D_{R}(p)$. The maximum principle applies and gives $E_{R}^{0} \leq E_{R}$ on $D_{R}(p)$.

When $\lambda=1$, using (2.3), (3.2), (3.3), (3.4) and provided that $\left\|\partial_{t}\right\|^{2} \leq 1$ and $h_{1}(r)=\frac{1}{\tan r}$ we have, on $D_{R}(p)$,

$$
\begin{aligned}
& \Delta^{P} E_{R}^{\lambda}=E_{R}^{\lambda^{\prime \prime}}(r)\left\|\partial_{t}\right\|^{2}+E_{R}^{\lambda^{\prime}}(r)\left\{-\frac{1}{\tan r}\left\|\partial_{t}\right\|^{2}+(n-1)\left(\frac{1}{\tan r}+\left\langle H, \partial_{r}\right\rangle\right)\right\} \\
& =\sin ^{2} r \mathcal{E}_{R}^{\lambda^{\prime \prime}}(s)\left\|\partial_{t}\right\|^{2}+(n-1) \frac{1}{\tan r} \sin r \mathcal{E}_{R}^{\lambda^{\prime}}(s)+(n-1) \sin r \mathcal{E}_{R}^{\lambda^{\prime}}(s)\left\langle H, \partial_{r}\right\rangle \\
& \geq \sin ^{2} r \mathcal{E}_{R}^{\lambda^{\prime \prime}}(s)+\cos r \mathcal{E}_{R}^{\lambda^{\prime}}(s)+\left(\frac{n-2}{\tan r}\right) \sin r \mathcal{E}_{R}^{\lambda^{\prime}}(s)+(n-1) \sin r \mathcal{E}_{R}^{\lambda^{\prime}}(s)\left\langle H, \partial_{r}\right\rangle \\
& =E_{R}^{\lambda^{\prime \prime}}(r)+E_{R}^{\lambda^{\prime}}(r)\left(\frac{n-2}{\tan r}\right)+(n-1) E_{R}^{\lambda^{\prime}}(r)\left\langle H, \partial_{r}\right\rangle \\
& =-1+(n-1) E_{R}^{\lambda^{\prime}}(r)\left\langle H, \partial_{r}\right\rangle .
\end{aligned}
$$

Now, the result follows as in the euclidean case, using (3.6).

If $E_{R}^{\lambda}=E_{R}$ on $D_{R}(p)$, then the above inequalities in (3.7) will be equalities, and therefore, $\left\langle H, \partial_{r}\right\rangle=0$ on $D_{R}(p)$.

Then, when $\lambda=0$, Theorem 3 in [Ma1] applies and, on account of the convexity of $P$, we have that $D_{R}(p)$ is totally geodesic, so $D_{R}(p)=D_{R}^{\lambda}$.

If $\lambda=1$, as $\mathcal{E}_{R}^{\lambda^{\prime \prime}}<0$, we conclude, from the way we obtained the inequality (3.8), that the equality implies

$$
\left\|\partial_{t}\right\|^{2}=\left\|\partial_{r}\right\|^{2}=1
$$

so $\left\langle\xi, \partial_{r}\right\rangle(q)=0 \forall q \in D_{R}(p)$. Then, the geodesics in $S^{n}(1)$ joining $p$ and the points $q \in D_{R}(p)$ lie in $D_{R}(p)$ and are tangent to $D_{R}(p)$ at $p$; hence, $D_{R}(p)$ is totally geodesic. In fact, $D_{R}(p)=\exp _{p} \widetilde{D}_{R}(p)$, where $\exp _{p}$ is the exponential map in $S^{n}(1)$ and $\widetilde{D}_{R}(p)$ is the disc of radius $R$ in $T_{p} D_{R}(p)$. Therefore, $D_{R}(p)=D_{R}^{\lambda}$. 


\section{REFERENCES}

[CGM] Carreras, F.J., Gimenez, F. and Miquel, V., Immersions of compact riemannian manifolds onto a ball in a complex space form, To appear in Math. Zeit.

[Ch] Chavel, I., Eigenvalues in Riemannian Geometry, Academic Press, New York, 1984. MR 86g:58140

[ChYL] Cheng, S.Y., Li,P. and Yau, S.T., Heat equations on minimal submanifolds and their applications, Amer. J. Math. 106 (1984), 1033-1065. MR 85m:58171

[DCW] Do Carmo, M. and Warner, F.W., Rigidity and convexity of hypersurfaces in spheres, J. Differential Geometry 4 (1970), 133-144. MR 42:1014

[Dy] Dynkin, E.B., Markov processes, Springer Verlag, 1965. MR 33:1887

[GW] Greene, R.E. and Wu, H., Function Theory on Manifolds Which Possess a Pole, SpringerVerlag, New York, 1979. MR 81a:53002

[JK] Jorge,L.P. and Koutroufiotis, D., An estimate for the curvature of bounded submanifolds, Amer. J. Math. 103 (1981), 711-725. MR 83d:53041b

[KN] Kobayashi S. and Nomizu K., Foundations of Differential Geometry, Interscience Publishers, New York, 1963. MR 27:2945

[KP1] Karp, L. and Pinsky, M., Mean exit time from an extrinsic ball, Pitman Research Notes in Math. 150 (1986), 179-186. MR 89e:60155

[KP2] Karp, L. and Pinsky, M., Volume of a small extrinsic ball in a submanifold, Bull. London Math. Soc. 21 (1989), 87-92. MR 89i:53038

[M] Mueller, C., A Counterexample for Brownian Motion on manifolds, Contemporary Mathematics 73 (1988), 217-221. MR 89k:58297

[Ma1] Markvorsen, S., On the mean exit time from a minimal submanifold, J. Diff. Geom. 29 (1989), 1-8. MR 90a:58190

[Ma2] Markvorsen, S., On the heat kernel comparison theorems for minimal submanifolds, Proc. Amer. Math. Soc. 97 (1986), 479-482. MR 87i:58160

[Sp] Spivak, M., A comprehensive introduction to Differential Geometry, Publish or Perish Inc., Houston, 1979. MR 82g:53003a

Departament de Matematiques, Universitat Jaume I, Castello, Spain

E-mail address: palmer@mat.uji.es 